Metal-Mediated

Synthesis

Key words

\section{Pd-Ligand Controlled Cyclization for the Preparation of Heteroaromatics}

heterocycles

cyclization

carbazoles

indoles

dibenzazepines

acridines

Synthesis of dibenzazepines:

Synthesis of acridines:

palladium<smiles>C1=Cc2ccccc2Nc2ccccc21</smiles>

- DavePhos (2.25 mol\%) $\mathrm{Pd}_{2}(\mathrm{dba})_{3}(0.75 \mathrm{~mol} \%)$ $\mathrm{NaOt}$-Bu (1.5 equiv)

7-endo $99 \%$ yield

Synthesis of carbazoles:<smiles>C=Cc1ccccc1Nc1ccccc1Cl</smiles>

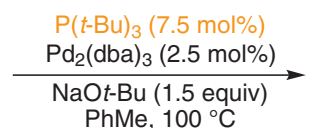<smiles>Cc1c2ccccc2nc2ccccc12</smiles>

6-exo $87 \%$ yield
Selected examples:<smiles>Cc1nc(C)c2c(n1)Nc1ccccc1C=C2</smiles>

$74 \%$ yield<smiles>C=Cc1cccc2c1[nH]c1ccccc12</smiles>

$94 \%$ yield<smiles>COc1cc2nc3cc(C)ccc3ccc-2cc1=O</smiles>

$83 \%$ yield<smiles>Cc1ccc2nc3ccccc3c(C)c2c1</smiles>

$98 \%$ yield

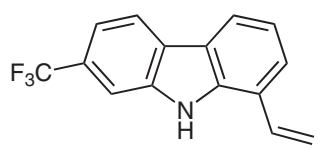

$89 \%$ yield

Synthesis of indoles:<smiles>C=Cc1ccccc1Nc1nc(C)nc(C)c1Cl</smiles><smiles>Cc1nc(C)c(Cl)c(-n2ccc3ccccc32)n1</smiles>

$91 \%$ yield

Significance: Various vinyl diphenylamine intermediates are selectively cyclized to the corresponding five-, six- or seven-membered heteroaromatics. The regioselectivity of these palladiumcatalyzed cyclizations is almost exclusively directed by the used ligand.

sYNFacts Contributors: Paul Knochel, Andreas K. Steib

Synfacts 2011, 1, 0075-0075 Published online: 21.12.2010 Dol: 10.1055/s-0030-1259114; Reg-No.: P15510SF
Comment: By using 2-bromostyrene and 2-chloroaniline derivatives in this palladium-catalyzed reaction with DavePhos as ligand, a direct onepot synthesis was achieved, furnishing $5 \mathrm{H}$-diazepines in good to excellent yields. 